\title{
A modified algebraic reconstruction algorithm for sparse projection
}

\author{
Hongyan $\mathrm{Li}^{1}$, Zhonglin Wan ${ }^{2}$ \\ ${ }^{1}$ School of Computer and Information, City College of Dongguan University of Technology, Dongguan, China; ${ }^{2}$ Department of Finance and \\ Economics, Dongguan Polytechnic, Dongguan, China \\ Contributions: (I) Conception and design: H Li; (II) Administrative support: Both authors; (III) Provision of study materials or patients: H Li; (IV) \\ Collection and assembly of data: H Li; (V) Data analysis and interpretation: Both authors; (VI) Manuscript writing: Both authors; (VII) Final \\ approval of manuscript: Both authors. \\ Correspondence to: Hongyan Li. School of Computer and Information, City College of Dongguan University of Technology, Dongguan, China. \\ Email: lihy@ccdgut.edu.cn.
}

Background: Computed tomography (CT) is an advanced medical imaging technology. The images obtained by CT are helpful for improving diagnostic accuracy. Currently, CT is widely used in clinical settings for diagnosis and health examinations. However, full angle CT scanning has the disadvantage of causing radiation damage to the human body. Sparse angle projection CT scanning is the most effective way to minimize this damage, but the quality of the reconstructed image is reduced. Therefore, it is important to improve the reconstructed image quality produced by sparse angle projection.

Methods: In this paper, we focused on the algebraic reconstruction algorithm. To reduce the accumulation of random noise, we formulated a modified algebraic reconstruction algorithm. Firstly, the algebraic reconstruction algorithm was used to compute two consecutive results, and then the weighted sum of these two results was used to correct the reconstructed image, and an iterative result was obtained. Using this method, we aimed to reduce the noise accumulation caused by iteration.

Results: In this study, 20 angle projections were used for the reconstruction. The experimental object was the Shepp-Logan phantom test image. The experiments were implemented under two conditions: without noise and with noise. The peak signal to noise ratio (PSNR) and the mean squared error (MSE) of the reconstructed image from projections without noise were 76.0896 and 0.0016 , respectively. The PSNR and MSE of the reconstructed image from projections with noise were 75.8263 and 0.0017 , respectively. The reconstructed performance was superior to the previous algebraic reconstruction algorithm.

Conclusions: The performance of the proposed method was superior to other algorithms, which confirms that noise accumulation caused by iteration can be effectively reduced by the weighted summation of two consecutive reconstruction results. Moreover, the reconstruction performance under noisy projection is superior to other algorithms, which demonstrates that the proposed method improves anti-noise performance.

Keywords: Computed tomography imaging (CT imaging); algebraic reconstruction algorithm; sparse projection

Submitted Jun 24, 2021. Accepted for publication Aug 05, 2021.

doi: $10.21037 /$ atm-21-3529

View this article at: https://dx.doi.org/10.21037/atm-21-3529

\section{Introduction}

CT imaging technology is the process of using X-ray to scan an object in a rotating projection, obtaining the projection data of the object from different angles, and then reconstructing the projection data into a digital image by computer processing. With the help of CT images, the size, shape, and pathological changes of human tissues and organs can be clearly observed. Therefore, CT imaging 
technology is vitally important for modern medical diagnosis. It assists doctors in diagnosing diseases and also improves the accuracy and objectivity of the diagnosis. In order to reconstruct clear CT images, the most direct and effective method is to increase the number of scanning projections. However, this causes increased $\mathrm{X}$-ray radiation to the human body, which is detrimental to health. Whilst full angle projection can restore high-quality images by using filtered background projection (FBP), in the case of incomplete projection data, such as sparse projection, the image reconstructed by FBP has serious artifacts due to the influence of noise. Therefore, image reconstruction with sparse angle or low dose has become the focus of more recent research $(1,2)$.

There are two methods for CT image reconstruction: the analytical FBP method and the iterative method. In the case of sparse projection data, the FBP algorithm fails. Compared with the FBP algorithm, the iterative reconstruction algorithm requires less projection data and can add different prior knowledge and constraints into the iterative process, so the iterative reconstruction algorithm is more suitable for sparse projection reconstruction problems. In the iterative method, the image is discretized firstly, that is, the unknown image to be reconstructed is discretized into an image grid. Then, according to the physical process of imaging and the corresponding mathematical model, the algebraic equations between the image to be reconstructed and the projection data are established, and the problem of image reconstruction can be transformed into the problem of solving linear equations. Usually, the linear equations are incompatible, so it cannot be solved by analytical method, only by iterative method. The iterative reconstruction algorithm sets the initial value to the reconstructed image and obtains the projection data from different angles. Then in each iteration, the error between the calculated results of projection and the measured data is used to correct the current estimated image. In this way, the projection calculated from the estimated image will be closer to the measured data, so as to obtain a more accurate image.

The iterative reconstruction algorithms used in CT reconstruction mainly include algebraic reconstruction methods and statistical reconstruction methods (3). Algebraic reconstruction algorithm is a typical reconstruction algorithm based on projections onto convex sets (POCS), which is easy to implement and has a certain anti-noise ability. Thus we focus on algebraic reconstruction algorithms. Currently there are many researches on algebraic reconstruction algorithms, such as algebraic reconstruction technology (ART) (4) and the simultaneous algebraic reconstruction technique (SART) (5). The main difference between ART and SART is that each correction of ART only considers one ray, and the reconstruction results are related to the order of the data used, while SART uses the correction values of all rays passing through a pixel to determine the average correction value of that pixel. When all the rays passing through the pixel are corrected, an iterative process is thus completed. In this way, the average correction can suppress some interference factors, and the calculation results are independent of the order of the data used.

In 2006, Donoho (6) proposed the compressed sensing (CS) theory. Sparse or compressible signals can be recovered by CS technology. With the development of compressed sensing theory, the algebraic iterative reconstruction theory based on total variation (TV) has made some progress in sparse angle projection reconstruction. Sidky and colleagues proposed an ASD-POCS (Adaptive steepest descent projection on convex sets) algorithm based on compressed sensing theory, which combined TV steepest descent and convex set projection constraints $(7,8)$. Lian proposed an algebraic iterative reconstruction algorithm, ART-TV, based on compressed sensing theory (9). In this method, after each iteration of ART, the gradient descent method was used to adjust the total variation. The ART-TV algorithm obtained a better image than ART with less projection data. Tai et al. proposed the SART-TV algorithm (10), which adaptively adjusts the step size of the gradient descent algorithm. Although this method improves the reconstruction speed, the reconstruction quality remains less than optimal. A limitation of all these methods is that they do not consider the random noise produced by iteration, which will make the iterative noise gradually accumulate, resulting in poor image quality.

For the algebraic reconstruction algorithm, the convergence speed has been a great concern. There are mainly two methods to improve the convergence speed: one is iterative formula, the other one is partition algorithm. In terms of iterative formula, SART method calculates the error between all estimated projection and measured values of one pixel, and then corrects the image. This improvement makes the convergence speed much faster than ART method. On the other hand, some scholars proposed to use partition algorithm to improve the convergence speed (11). With the rapid development of modern computer hardware, the iterative convergence speed has been gradually ignored. This paper focuses on the random noise of iterative algorithm itself, which is a significant 
problem in sparse projection reconstruction.

In this paper, a modified SART-TV algorithm is proposed in view of the significant random noise created by sparse angle projection. Using the results of two adjacent iterations, a new correction term is constructed to alleviate the noise accumulation in the reconstruction model and further enhance the anti-noise ability. Experimental results verify the effectiveness of the improved algorithm. We present the following article in accordance with the MDAR reporting checklist (available at https://dx.doi.org/10.21037/ atm-21-3529).

\section{Methods}

\section{Related work}

In the algebraic reconstruction algorithm, the image should be discretized first. Then the relationship between the pixels and projection data can be expressed by linear equations, and the reconstruction image can be regarded as the solution of linear equations. The equations can be written in matrix form as in Eq. [1].

$$
A X=P
$$

Where, $X=\left[x_{1}, x_{2} \cdots, x_{N^{2}}\right], P=\left[p_{1}, p_{2} \cdots, p_{M}\right], N \times N$ is the image size, $M$ is the number of projection data, $x_{j}(j=1,2, \cdots, N \times N)$ is the image pixel value, $p_{i}(i=1,2, \cdots, M)$ is the projection value. The radiation passing through the object is detected and recorded by the detector after attenuation. According to Beer's Law, the projection value recorded by the detector is equivalent to the sum of the linear attenuation coefficients of the object on the path through which the ray passes (9). Therefore, Eq. [1] is equivalent to Eq. [2].

$$
\sum_{j} a_{i j} x_{j}=p_{i}
$$

Where, the element of matrix $\mathrm{A}$ is the contribution of the $j$ th pixel to the $i$ th detector unit. Using various iterative algorithms to solve the linear equations, $X$ is the pixel value of the image to be reconstructed.

In CT scan imaging, matrix $A$ is very large and not full rank. Generally, it is not a square matrix. In addition, the projection data is noisy, so the equations are incompatible. Many of the traditional methods for solving linear equations cannot be used. The commonly used iterative methods are ART and SART.

The iterative formula of the SART algorithm is as follows:

$$
\forall j: x_{j}^{k+1}=x_{j}^{k}+\lambda \frac{\sum_{i \in I_{\theta}} a_{i j} \frac{p_{i}-\sum_{m=1}^{M} a_{i m} x_{m}}{\sum_{m=1}^{M} a_{i m}}}{\sum_{i \in I_{\theta}} a_{i j}}
$$

Where $k$ is the number of iterations, $1 \leq i \leq N, 1 \leq \mathrm{j} \leq \mathrm{M}$ and $\lambda$ is the relaxation factor, and $I_{\theta}$ is the projection index set under a projection angle.

The number of sub-iterations in the SART algorithm is much less than that of the ART algorithm in every iteration process, but the image reconstructed by SART is smoother than that reconstructed by ART and can better suppress the stripe artifacts. In complete projection reconstruction, the algebraic reconstruction algorithm was initially considered too difficult to use due to the large amount of computation needed and the large memory requirements, but with the rapid development of computer technology, these original drawbacks have now been resolved. Therefore, the algebraic reconstruction algorithm has more recently begun to play a central role in sparse angle projection reconstruction.

In sparse angle projection, the compressed sensing principle is introduced to reconstruct the image with a small amount of projection data. Generally the CT image itself is not sparse, but the gradient of the image is sparse. Therefore, the L1 norm of the image gradient, i.e., the TV, is generally selected as the sparse transformation of the image. The TV algorithm model of sparse reconstruction is:

$$
\min _{X}|| X||_{\mathrm{TV}}, \text { s.t. } A X=P
$$

In reference (10), a SART-TV algorithm is proposed, which combines SART with a TV algorithm. When an iteration of SART is completed, the gradient descent method is applied to minimize the TV norm of the image, and the TV convergence step size is dynamically adjusted. Although the convergence rate is faster, the quality of the sparse projection reconstruction still needs improvement.

\section{Proposed method}

The traditional SART algorithm does not consider the shortage of data and the severity of random noise in the sparse projection reconstruction, so the reconstruction accuracy under the sparse angle requires improvement. In this paper, a modified SART-TV algorithm is proposed, which takes into consideration that the pixel values generated by each iteration will include some random noises. 
Firstly, a random correction term is introduced to modify the SART iteration for the second time to alleviate the accumulation of errors in the iteration cycle and improve the anti-noise ability. The random correction term is weighted by the iterative result of the previous algorithm and the SART iteration result, and the weight factor is generated by the normalization of the difference between the two iteration results. The modification is completed by Eq. [7] without introducing additional parameters and increasing the difficulty of the parameter adjustment.

$$
\begin{aligned}
& X^{(k+1)}=\operatorname{SART}\left(X^{(k)}\right) \\
& e=\frac{X^{(k+1)}-X^{(k)}}{\left\|X^{(k+1)}-X^{(k)}\right\|^{2}} \\
& X^{(k+1)}=X^{(k+1)}+e \cdot X^{(k)}
\end{aligned}
$$

Secondly, sparse reconstruction is carried out with the TV algorithm to deal with the reconstruction noise caused by insufficient data.

$$
\begin{aligned}
& \forall j: X_{j}^{T V}=\frac{\partial\left\|X_{j}^{(k+1)}\right\|_{T V}}{\partial X_{j}^{(k+1)}} \\
& X^{(k+1)}=X^{(k+1)}-\alpha \cdot X^{T V}
\end{aligned}
$$

Among them $\alpha$ is the step size of gradient descent, and the adaptive adjustment is completed according to reference (10).

The proposed algorithm is as follows:

$$
\begin{aligned}
& \text { 1: Initiate } X^{(1)}=0, N_{\text {iter }}, N_{\text {iter } 2}, \lambda \text {; } \\
& \text { 2: for } k=1,2, \ldots, N_{i t e r l} \text {; } \\
& \text { 3: } X^{\text {old }}=X^{(\mathrm{k})} \text {; } \\
& \text { 4: } X^{\text {new }}=\operatorname{SART}\left(X^{\text {old }}\right) \text {; }
\end{aligned}
$$

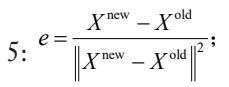

$$
\begin{aligned}
& \text { 6: } X^{\mathrm{new}}=X^{\mathrm{new}}+e^{*} X^{\text {old }} \text {; } \\
& \text { 7: all } j: X_{j}^{\text {vv }}=\frac{\partial\left\|X_{j}^{\text {new }}\right\|_{T V}}{\partial X_{j}^{\text {new }}} ; \\
& \text { 8: for } i=1: N_{\text {iter } 2 \text {; }} \\
& \text { 9: } \quad X^{\text {new }}=X^{\text {new }}-\alpha^{*} X^{t v} \text {; } \\
& \text { 10: end for ; } \\
& \text { 11: } X^{(k+1)}=X^{\text {new }} \text {; } \\
& \text { 12: end for ; }
\end{aligned}
$$

\section{Results}

\section{Evaluation criteria}

In this paper, the Shepp-Logan phantom test image was used to simulate the reconstruction process. All experiments were implemented by Matlab2017a. We compared the proposed method with ART, SART, and SART-TV. In order to verify the anti-noise performance of each algorithm, Gaussian noise with an average value of 0 and a standard deviation of 0.2 was added to the normalized projection sine diagram of the Shepp-Logan phantom. In the experiments, we took the relaxation factor $\lambda$ as 0.0002 and compared the original image with the reconstructed image to judge whether the reconstruction result was good or bad. In order to quantitatively describe the reconstructed image quality of the four reconstruction methods with or without noise, the mean square error (MSE) and peak signal to noise ratio (PSNR) were used to measure the quality of the reconstructed images. The formula is as follows:

$$
\begin{aligned}
& M S E=\frac{1}{N \cdot N} \sum_{j}\left\|f_{j}-\hat{f}_{j}\right\|^{2} \\
& P S N R=10 \times \log _{10}\left(\frac{M A X_{I}^{2}}{M S E}\right)
\end{aligned}
$$

In Eq. [11], $M A X_{\mathrm{I}}$ represents the maximum value of the image color, and 8-bit sampling points are expressed as 255. MSE is the mean square error between the original image and the reconstructed image. The smaller the MSE is, the smaller the pixel gray difference between the reconstructed image and the real image is, and the higher the reconstruction quality. PSNR is the ratio of the maximum possible power of a signal to the destructive noise power that affects its representation accuracy. The higher the PSNR is, the smaller the distortion of the image is, in decibels $(\mathrm{db})$.

\section{Experimental studies}

In our experiments, the reconstruction results from 20 projection angles were compared. There were 256 ray beams at each angle. The number of iterations was set to 300. Figure 1 shows the reconstruction results of each algorithm without noise. The image reconstructed by the proposed method is clearer and less noisy than the other methods. The PSNR and MSE of the corresponding images are shown in Table 1. The PSNR and MSE of the 

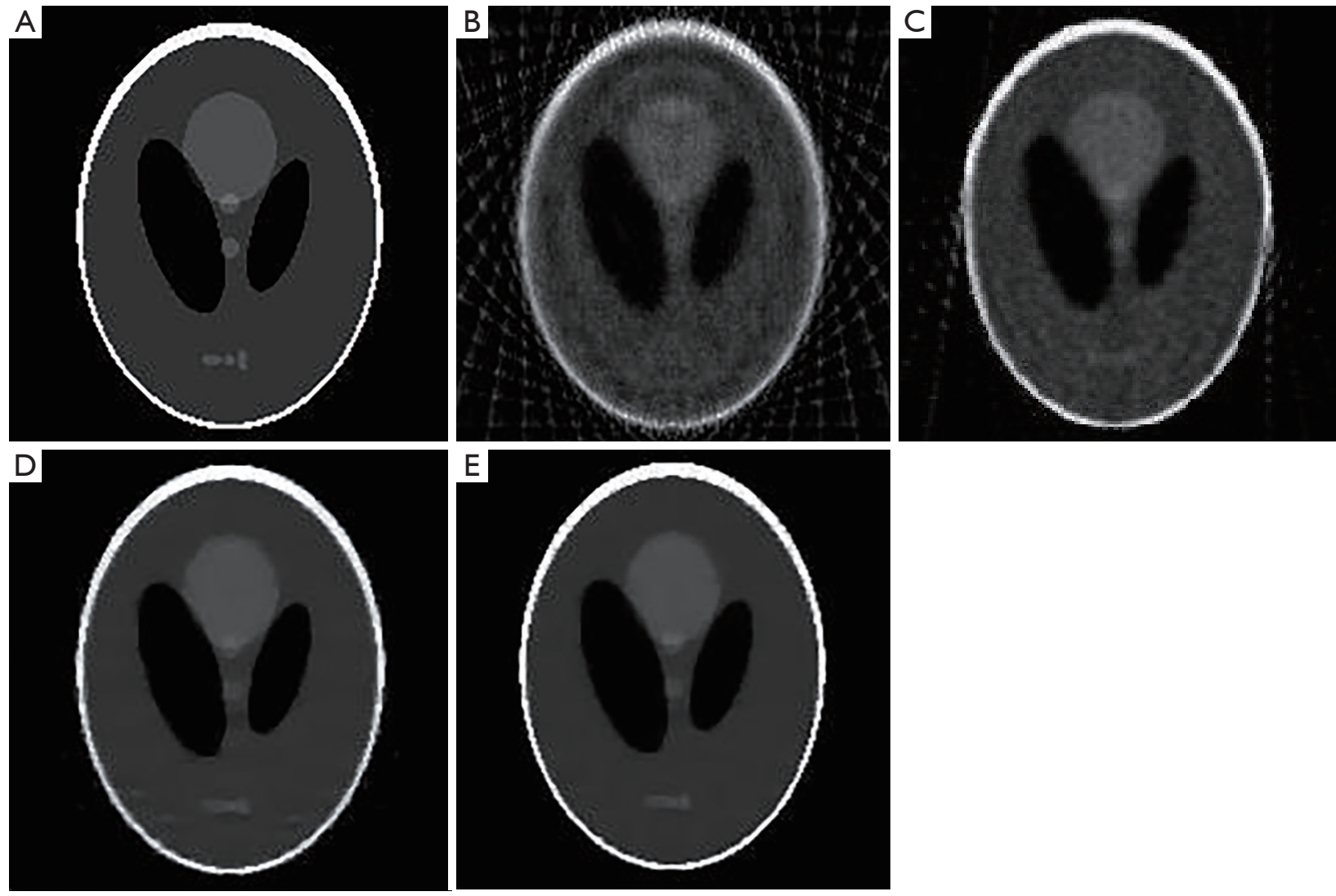

Figure 1 The reconstruction images without noisy projections. (A) Shepp-Logan phantom; (B) ART; (C) SART; (D) SART-TV; (E) Proposed algorithm. ART, algebraic reconstruction technology; SART, simultaneous algebraic reconstruction technique; TV, total variation.

Table 1 The MSE and PSNR of the reconstructed images without noisy projections

\begin{tabular}{lcc}
\hline Algorithms & PSNR & MSE \\
\hline ART & 63.6129 & 0.0283 \\
SART & 66.5472 & 0.0144 \\
SART-TV & 72.9457 & 0.0033 \\
Proposed method & 76.0896 & 0.0016 \\
\hline
\end{tabular}

MSE, mean squared error; PSNR, peak signal to noise ratio; ART, algebraic reconstruction technology; SART, simultaneous algebraic reconstruction technique; TV, total variation.

image reconstructed by the proposed method are 76.0896 and 0.0016 , respectively. The reconstruction results under the condition of noisy projections are shown in Figure 2 and Table 2. The image reconstructed by the proposed method is closer to the original than the other images. And the PSNR and MSE of the image reconstructed by the proposed method are 75.8263 and 0.0017 , respectively.

Compared with the SART algorithm, the PSNR of the proposed algorithm is $9.54 \mathrm{db}$ higher without noise and $9.79 \mathrm{db}$ higher under noisy projection. Compared with the SART-TV algorithm, PSNR is $3.14 \mathrm{db}$ higher in the noiseless condition and $3.14 \mathrm{db}$ higher in the noisy projection.

\section{Discussion}

In sparse projection reconstructions, ART produces a lot of stripe artifacts, and the SART algorithm also has some artifacts. However, the reconstruction results of the modified SART-TV algorithm based on compressed sensing have been significantly improved, and the image reconstructed by the proposed algorithm is better than that of the original SART-TV algorithm, which demonstrates that the modified algorithm is effective in reducing noise and optimizing the reconstructed image. 

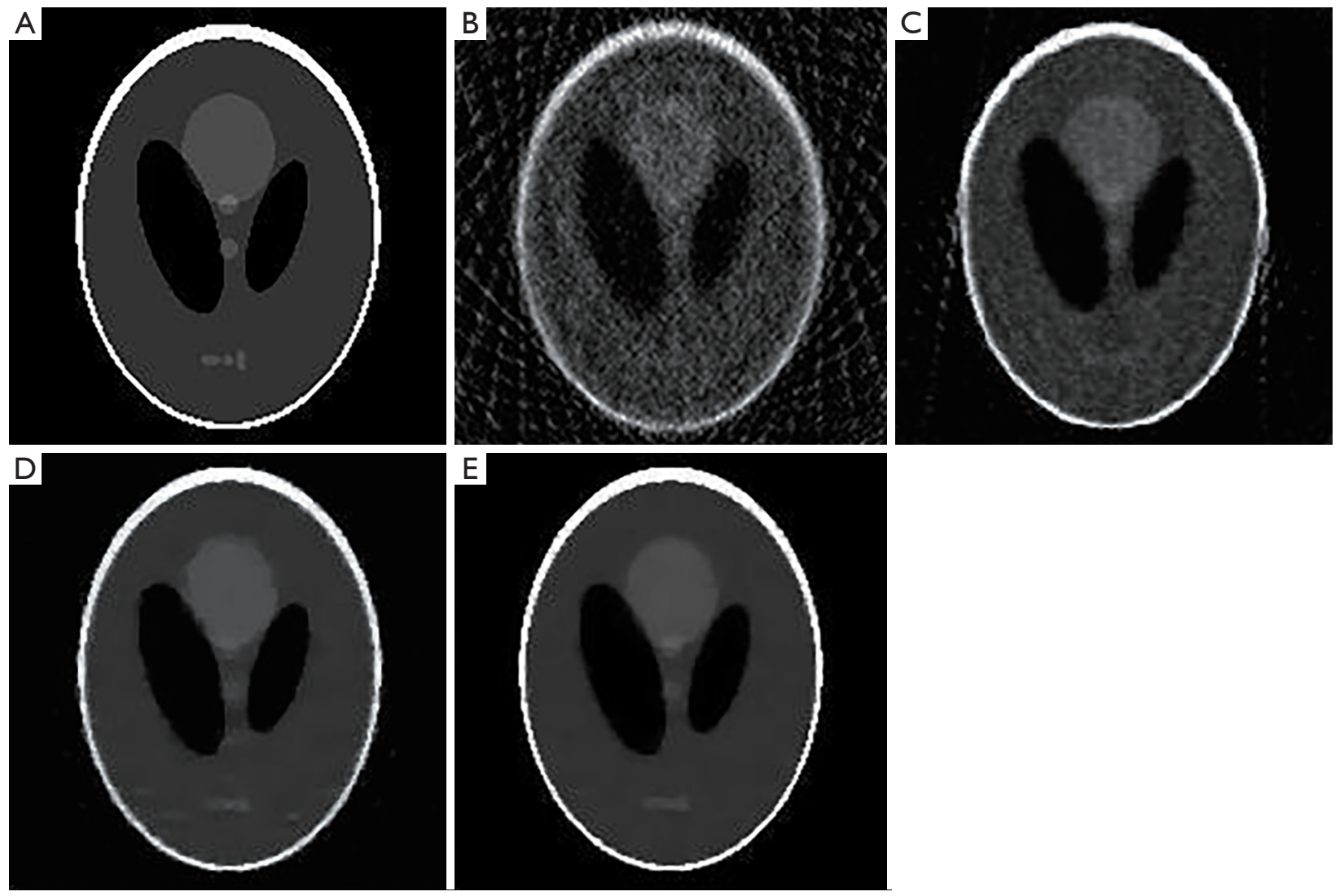

Figure 2 The reconstruction images with noisy projections. (A) Shepp-Logan phantom; (B) ART; (C) SART; (D) SART-TV; (E) Proposed algorithm. ART, algebraic reconstruction technology; SART, simultaneous algebraic reconstruction technique; TV, total variation.

Table 2 The MSE and PSNR of the reconstructed images with noisy projections

\begin{tabular}{lcc}
\hline Algorithms & PSNR & MSE \\
\hline ART & 63.3451 & 0.0301 \\
SART & 66.0357 & 0.0162 \\
SART-TV & 72.6901 & 0.0035 \\
Proposed method & 75.8263 & 0.0017 \\
\hline
\end{tabular}

MSE, mean squared error; PSNR, peak signal to noise ratio; ART, algebraic reconstruction technology; SART, simultaneous algebraic reconstruction technique; TV, total variation.

Due to the random noise and the serious shortage of data, the signal-to-noise ratio (SNR) of low-projection CT imaging systems is very low. A sparse projection CT image reconstruction algorithm should have a strong anti-noise performance to obtain more details of the reconstructed image. According to the results of Figure 2, the overall gap between the reconstructed image and the standard SheppLogan phantom is the smallest, and it has high stability and anti-noise ability.

With or without noise, the MSE of the proposed method is the smallest, and the PSNR index is the largest, which is enough to show that the reconstruction quality is the highest. Table 2 shows that the proposed algorithm can reconstruct images with a higher quality than SART and SART-TV under sparse projection. In addition, each algorithm has a better ability to resist noise. In particular, the PSNR and MSE of the proposed method in this paper show little change with noisy projections. Therefore, it is proved that the proposed method has good anti-noise performance and can reconstruct high-quality CT images with less projection.

To solve the problem of severe random noise in CT image reconstruction with few projections, we proposed an improved SART-TV algorithm to reconstruct the sparse projections of the Shepp-Logan phantom. Our experimental 
results demonstrate that the proposed method is superior to other algorithms. Our results also verify that the noise accumulation caused by iteration can be effectively reduced by weighted summation of two consecutive reconstruction results. Moreover, the reconstruction performance under noisy projection is superior to other algorithms, which demonstrates that the proposed method improves anti-noise performance.

Although the performance of proposed method is better than previous algorithms, there are still some smoothing problems in the reconstructed image, some edges are relatively fuzzy. Thus, the method to deal with smoothing problems will be future work.

\section{Acknowledgments}

Funding: This work is supported by the Youth Innovative Talents Project of Guangdong Province (No: 2017KQNCX55).

\section{Footnote}

Reporting Checklist: The authors have completed the MDAR reporting checklist. Available at https://dx.doi. org/10.21037/atm-21-3529

Data Sharing Statement: Available at https://dx.doi. org/10.21037/atm-21-3529

Conflicts of Interest: Both authors have completed the ICMJE uniform disclosure form (available at https://dx.doi. org/10.21037/atm-21-3529). The authors report funding support from the Youth Innovative Talents Project of Guangdong Province (No: 2017KQNCX55). The authors have no other conflicts of interest to declare.

Ethical Statement: The authors are accountable for all aspects of the work in ensuring that questions related to the accuracy or integrity of any part of the work are appropriately investigated and resolved.

Open Access Statement: This is an Open Access article distributed in accordance with the Creative Commons Attribution-NonCommercial-NoDerivs 4.0 International License (CC BY-NC-ND 4.0), which permits the noncommercial replication and distribution of the article with the strict proviso that no changes or edits are made and the original work is properly cited (including links to both the formal publication through the relevant DOI and the license).
See: https://creativecommons.org/licenses/by-nc-nd/4.0/.

\section{References}

1. Gunn ML, Kohr JR. State of the art: technologies for computed tomography dose reduction. Emerg Radiol 2010;17:209-18.

2. Wang G, Yu H, Ye Y. A scheme for multisource interior tomography. Med Phys 2009;36:3575-81.

3. Silva AC, Lawder HJ, Hara A, Kujak J, Pavlicek W. Innovations in CT dose reduction strategy: application of the adaptive statistical iterative reconstruction algorithm.AJR Am J Roentgenol 2010;194:191-9.

4. Gordon R, Bender R, Herman GT. Algebraic reconstruction techniques (ART) for three-dimensional electron microscopy and x-ray photography. J Theor Biol 1970;29:471-81.

5. Andersen AH, Kak AC. Simultaneous algebraic reconstruction technique (SART): a superior implementation of the art algorithm. Ultrason Imaging 1984;6:81-94.

6. Donoho DL. Compressed sensing. IEEE Transactions on Information Theory 2006;52:1289-306.

7. Sidky EY, Kao CM, Pan X. Accurate image reconstruction from few-views and limited-angle data in divergentbeam CT(J). Journal of X-ray Science and Technology 2006;14:119-39.

8. Sidky EY, Pan X. Image reconstruction in circular cone-beam computed tomography by constrained, total-variation minimization. Phys Med Biol 2008;53:4777-807.

9. Lian QS, Hao PP. CT image reconstruction based on compressed sensing and algebraic reconstruction. Optical Technology 2009;35:422-5.

10. Tai HW, Qiao ZW. GPU gradient adaptive SART image reconstruction algorithm. Nuclear Electronics and Detection Technology 2016;36:877-9.

11. Wang G, Jiang M. Ordered-subset simultaneous algebraic reconstruction techniques (OS-SART). Journal of X-Ray Science and Technology 2004;12:169-177.

(English Language Editor: D. Fitzgerald)

Cite this article as: $\mathrm{Li} \mathrm{H}$, Wan $\mathrm{Z}$. A modified algebraic reconstruction algorithm for sparse projection. Ann Transl Med 2021;9(18):1422. doi: 10.21037/atm-21-3529 